\title{
Autorialità, genere e sistema letterario: conversazioni con Antonella Cilento, Helena Janeczek, Laura Pugno, Caterina Serra e Nadia Terranova
}

Auctorialité, genre et système littéraire : entretiens avec Antonella Cilento, Helena Janeczek, Laura Pugno, Caterina Serra et Nadia Terranova Authorship, Gender and the Literary System: Conversations with Antonella Cilento, Helena Janeczek, Laura Pugno, Caterina Serra and Nadia Terranova

Alberica Bazzoni

\section{(2) OpenEdition}

\section{Journals}

Edizione digitale

URL: https://journals.openedition.org/cei/8532

DOI: 10.4000/cei.8532

ISSN: 2260-779X

Editore

UGA Éditions/Université Grenoble Alpes

Edizione cartacea

ISBN: 978-2-37747-257-4

ISSN: 1770-9571

Notizia bibliografica digitale

Alberica Bazzoni, «Autorialità, genere e sistema letterario: conversazioni con Antonella Cilento, Helena Janeczek, Laura Pugno, Caterina Serra e Nadia Terranova», Cahiers d'études italiennes [Online], 32 | 2021, online dal 01 mars 2021, consultato il 16 septembre 2021. URL: http://journals.openedition.org/ cei/8532 ; DOl: https://doi.org/10.4000/cei.8532

Questo documento è stato generato automaticamente il 16 septembre 2021.

(c) ELLUG 


\title{
Autorialità, genere e sistema letterario: conversazioni con Antonella Cilento, Helena Janeczek, Laura Pugno, Caterina Serra e Nadia Terranova
}

\author{
Auctorialité, genre et système littéraire : entretiens avec Antonella Cilento, \\ Helena Janeczek, Laura Pugno, Caterina Serra et Nadia Terranova \\ Authorship, Gender and the Literary System: Conversations with Antonella \\ Cilento, Helena Janeczek, Laura Pugno, Caterina Serra and Nadia Terranova
}

Alberica Bazzoni

\section{Introduzione}

1 L'articolo esplora il sistema letterario italiano da un punto di vista di genere, attraverso lo sguardo di cinque scrittrici contemporanee: Antonella Cilento, Helena Janeczek, Laura Pugno, Caterina Serra e Nadia Terranova ${ }^{1}$. Le interviste, realizzate nel corso del 2018 e 2019, consentono una duplice focalizzazione: da un lato, mirano a far emergere le dinamiche di genere nel campo della letteratura italiana contemporanea secondo la prospettiva interna di chi vi partecipa da vicino; dall'altro, danno voce al posizionamento soggettivo e all'esperienza delle scrittrici stesse, nell'intreccio con la loro personale poetica e postura politica. Le conversazioni ruotano intorno a quattro aree tematiche principali: l'autorialità; le genealogie e il canone; il rapporto con l'editoria, la critica e il pubblico; il rapporto con il femminismo.

2 Prima di addentrarci nel corpo vivo delle interviste, è utile esplicitare alcune informazioni di contesto e alcune considerazioni di metodo e di carattere teorico che inquadrano questa ricerca nel suo complesso. L'approccio adottato in questa ricerca si 
rifà a una lunga tradizione di pensiero critico (di stampo marxista, femminista, postcoloniale, nero e queer) volto a decostruire le strutture di potere patriarcali, razziste, classiste ed eteronormative che permeano la produzione e la ricezione del discorso e impongono la naturalizzazione di rapporti di forza fondati sul genere, la razza, l'orientamento sessuale e la classe, e a smascherare così il falso universalismo del soggetto dominante e delle strutture di pensiero binarie ed essenzialiste di cui esso è portatore ${ }^{2}$. Come scrive Monique Wittig: «Il concetto di differenza non ha nulla di ontologico in sé. Si tratta semplicemente del modo in cui i padroni interpretano una situazione storica di dominio» ${ }^{3}$. In linea con tale approccio critico, come le conversazioni con le autrici mettono in rilievo, il termine 'donna' non è inteso qui in senso essenzialistico, né si postula alcun nesso di tipo deterministico fra genere e scrittura ${ }^{4}$. Allo stesso modo, non si considera il genere l'unico asse di analisi rilevante, bensì una dimensione messa specificamente a fuoco che interagisce allo stesso tempo con altri assi identitari, secondo una prospettiva intersezionale ${ }^{5}$. Le interviste sono infatti strutturate in modo tale da far emergere il posizionamento soggettivo delle scrittrici rispetto alla dimensione di genere, incluso se, quando e quanto sentano la dimensione di genere in senso generale e la propria identità sessuata come rilevanti nella propria attività artistica.

3 Le interviste discusse in questo articolo si inseriscono in un progetto di ricerca più ampio, volto ad investigare i meccanismi di potere e le assiologie valoriali che contribuiscono a riprodurre le gerarchie di genere allinterno del campo artistico italiano, e specificamente di quello letterario ${ }^{6}$. L'analisi qui proposta va ad interloquire con le numerose e variegate prese di posizione sul tema del genere nella letteratura che in questi anni sono emerse soprattutto nell'ambito di giornali e riviste ${ }^{7}$, suscitando un dibattito ad oggi tutto fuorché risolto o pacificato ma che stenta tuttavia a raggiungere ed intaccare il contesto universitario italiano, nonostante oramai oltre quarant'anni di studi teorici ed empirici femministi ${ }^{8}$. Rispetto agli studi esistenti, questa ricerca contribuisce una prospettiva inedita, quella delle autrici stesse, chiamate a rispondere sia in quanto artiste che in quanto agenti culturali. L'articolo mostra quindi la vitalità del tema del rapporto fra genere e scrittura e la pluralità delle risposte, interpretazioni e negoziazioni messe in campo dalle scrittrici intervistate.

Guardando al panorama letterario italiano contemporaneo si riscontra una marcata differenza di genere. Le scrittrici del passato, incluse quelle dell'Ottocento e del Novecento, sono per la maggior parte escluse o marginalizzate nelle storie letterarie, nei manuali e nei corsi universitari, e una situazione analoga si riproduce per quanto riguarda le scrittrici contemporanee, le quali si trovano ad affrontare forme sottili e però pervasive di marginalizzazione da parte della critica e dell'accademia letterarie italiane. Benché il numero di autrici e autori in Italia sia oggi pressoché pari, e così il rispettivo successo di lettura ${ }^{9}$, lo spazio della critica - cattedre universitarie, giurie di concorsi, ruoli dirigenziali nelle case editrici, saggistica, direzione di giornali, critica culturale - è ancora ampiamente dominato da uomini. In questo contesto, le scrittrici ottengono sistematicamente meno spazio rispetto agli scrittori nelle recensioni e nei festival, e ricevono un numero significativamente inferiore di premi letterari. Se guardiamo per esempio al Premio Strega, ad oggi il premio più prestigioso in Italia, la disparità di trattamento è palese:

- Premio Strega: Attivo dal 1947. Numero totale di premi conferiti: 72.

- Uomini: 61 (85\%); Donne: 11 (15\%). 
- Ultimi 10 anni (2010-2019): Uomini: 9; Donne: 1.

5 La vittoria dello Strega da parte di Helena Janeczek con La ragazza con la leica nel 2018 arriva dopo quindici anni consecutivi in cui il premio è stato assegnato a uno scrittore, ovvero dal 2003, quando l'ha vinto Melania Mazzucco con Vita. La vittoria di Janeczek è stata seguita nuovamente da un altro vincitore uomo nel 2019, Antonio Scurati con M. Il figlio del secolo. Fra i dodici finalisti del 2020 si contano nove uomini e tre donne, riconfermando così la stessa tendenza. La sproporzione è la stessa per quanto riguarda il premio Viareggio, mentre si raggiunge una situazione di relativo equilibrio nel premio Campiello, dove, a differenza degli altri premi, fattore decisivo è la presenza della giuria popolare (specificità che segnala quindi una differenza fra giudizio della critica e giudizio del pubblico $)^{10}$.

6 Nel complesso, la critica e l'accademia italiane mostrano una resistenza diffusa a prendere atto della disparità esistente, ovvero a interrogarne le ragioni sottese, i valori e i pregiudizi inconsci che orientano in primo luogo le scelte di lettura e, in secondo luogo, il riconoscimento di valore. Invece di rendere conto dei criteri e delle posizioni di potere che producono le scelte di merito, la critica italiana tende ancora a rivendicarne la neutralità e l'oggettività, $o$, in alternativa, ad appellarsi alla libertà idiosincratica del gusto personale, proiettando l'accusa di parzialità sui discorsi che criticano la cultura patriarcale di cui è permeato il mondo letterario non meno di quanto lo sia la società in senso ampio, e affermando così implicitamente - ma senza che sia mai dichiarato apertamente - un criterio semplice: i libri degli uomini sono migliori di quelli delle donne. Emblematica in questo senso la selezione operata da Andrea Cortellessa nel recente intervento «Del XXI secolo il canone è questo» ${ }^{11}$. Su una lista di 50 nomi che caratterizzerebbero i primi vent'anni di questo secolo, Cortellessa propone 45 scrittori e 5 scrittrici, una proporzione che non è evidentemente in alcun modo rappresentativa del panorama letterario italiano (metodologicamente difficile da argomentare l'esclusione di Elena Ferrante, ma anche quella di Melania Mazzucco, Donatella Di Pietrantonio, Simona Vinci, Patrizia Valduga, Patrizia Cavalli, solo per fare alcuni nomi) e che pure ha lo spazio, culturale e di potere, per imporsi come autorevole. Ovviamente, e questo è il punto critico cruciale, una certa selezione di testi produrrà un certo discorso critico, una certa narrazione su quella che è la letteratura italiana del '900 e contemporanea, una certa ricostruzione dei temi, delle innovazioni o stanchezze, del linguaggio, delle tensioni che la caratterizzano. Ignorare ed escludere i contributi delle scrittrici e fondare le proprie analisi solo o in larga maggioranza sui testi degli scrittori non ha quindi solo una ripercussione di tipo politico, nel senso di mettere in atto una discriminazione, ma anche sul piano metodologico ed ermeneutico, in quanto produce una lettura fortemente parziale della realtà, una parzialità che allo stesso tempo viene imposta come oggettiva e universale ${ }^{12}$.

7 In questo quadro critico complessivo, si inseriscono numerose iniziative collettive in campo editoriale e accademico volte a modificare le dinamiche invalse, diffondendo e valorizzando le opere delle scrittrici. Fra queste, particolare rilievo hanno i festival: Feminism: Fiera dell'editoria delle donne, che si svolge annualmente alla Casa internazionale delle donne di Roma a partire dal 2018; InQuiete: festival di scrittrici a Roma, dal 2017; Scrittrici insieme - Festival di letteratura al femminile, a Somma Lombardo dal 2011; Women's Fiction Festival - Festival internazionale di narrativa femminile, a Matera dal 2008. Un ruolo importante è svolto delle case editrici, come la storica La Tartaruga e, più di recente, L'iguana. Feminist Publishing; Il dito e la luna; Somara!Edizioni, a cui si 
affianca l'attività culturale delle librerie specializzate, come la storica Libreria delle donne di Milano e, oggi, la libreria Tuba di Roma, promotrice di un programma fittissimo di incontri e presentazioni, e la Società italiana delle letterate (compagna della Società italiana delle storiche e di IAPh Italia, sezione dell'Associazione internazionale delle filosofe) che svolge dal 1995 un'importante attività di riconnessione fra ricerca accademica e divulgazione. Infine, in assenza di dipartimenti di studi di genere e nel contesto fortemente conservatore della disciplina letteraria in Italia, a partire dagli anni '70 è andato crescendo il numero di studi che indagano il contributo delle scrittrici italiane e sviluppano una riflessione critica femminista sui fondamenti epistemologici del sapere, spesso in dialogo con la ricerca svolta dall'italianistica in altri paesi (e specialmente in ambito anglo-americano). L'attività combinata di queste reti e azioni culturali ha consentito e consente ancora oggi alle scrittrici di trovare uno spazio di valorizzazione, che passa dalla ristampa di testi fuori catalogo alla promozione di libri tramite presentazioni e recensioni, alla critica letteraria in senso stretto e alla trasformazione progressiva dei manuali e dei programmi scolastici e universitari.

\section{Le autrici}

8 Le scrittrici intervistate hanno in comune il fatto di affiancare la scrittura a una partecipazione attiva al mondo culturale, artistico e politico italiano in vari modi e attraverso vari canali, che vanno dalla collaborazione con giornali e riviste all'insegnamento di corsi di scrittura, all'organizzazione di festival ed eventi culturali, al coinvolgimento diretto in manifestazioni e azioni politiche, al lavoro in altri settori artistici come il cinema, la danza e le arti visive. In altre parole, sono tutte figure di intellettuali e agenti culturali, oltreché di scrittrici. Inoltre, appartengono tutte alla stessa generazione, nata tra la fine degli anni '60 e gli anni '70, e non hanno quindi vissuto in prima persona il femminismo della seconda ondata, formandosi in un clima culturale regressivo che dipingeva la parità fra i sessi come raggiunta e il femminismo come superato. Infine, ad eccezione di Helena Janeczeck, tedesca di origini ebree, le scrittrici intervistate sono bianche e native italiane, provenienti da diverse aree geografiche del Paese.

9 Antonella Cilento, nata a Napoli nel 1970, è autrice di numerosi romanzi e racconti, fra cui Non leggerai (Giunti, 2019); Morfisa o L'acqua che dorme (Mondadori, 2018); Lisario o il piacere infinito delle donne (Mondadori, 2014), finalista del premio Strega e vincitore del Premio Boccaccio, tradotto in Spagna, Francia, Germania, Corea, Lituania, Finlandia; Una lunga notte (Guanda, 2002), vincitore dei premi Fiesole e Viadana. Ha scritto per il teatro e per il cinema e collabora con numerosi quotidiani e riviste. Fra le diverse attività culturali che porta avanti, insegna scrittura creativa e cura la rassegna letteraria internazionale "Strane coppie". La sua scrittura, fortemente influenzata dal barocco e dalle arti visive e molto legata alla città di Napoli, spazia dalla rievocazione del passato, e spesso di un passato fantastico come in Lisario e in Morfisa, alla distopia futura in Non leggerai, al realismo sociale in La madonna dei mandarini (NN Editore, 2015).

Helena Janeczek è nata a Monaco di Baviera nel 1964 da una famiglia ebrea di origini polacche e vive in Italia, a Gallarate (Milano) dai primi anni '80, quando ha scelto di adottare l'italiano come propria lingua di espressione creativa. È autrice di Lezioni di tenebra (Mondadori, 1997 - Nuova ed. Guanda, 2011); Cibo (Mondadori, 2002); Le rondini di Montecassino (Guanda, 2010); Bloody Cow (Il Saggiatore, 2012) e La ragazza con la Leica 
(Guanda, 2017), vincitore del Premio Strega e del Premio Bagutta, tradotto in inglese da Ann Goldstein. Collabora con Nazione Indiana, Nuovi Argomenti e numerose testate nazionali fra cui Repubblica, Il Sole 24 Ore e L'Unità, ha lavorato come consulente editoriale per la narrativa straniera e organizza annualmente il festival SI Scrittrici Insieme. I suoi romanzi mescolano spesso autobiografia, documento storico e finzione, ed esplorano temi quali l'identità individuale e collettiva, l'amicizia, la memoria, la guerra e l'Olocausto.

11 Laura Pugno, nata a Roma nel 1970, ha vissuto in diversi Paesi europei per poi stabilirsi a Madrid, dove dal 2015 dirige l'Istituto Italiano di Cultura. A ciò accompagna un'intensa attività di traduttrice dall'inglese e dal francese e di collaboratrice radio (Radio Rai 3, Rai Educational) e a varie testate fra cui L'Espresso, Repubblica Roma e Il Manifesto. Ha creato il Premio Solinas Italia-Spagna per la sceneggiatura. Ha pubblicato romanzi, poesie, racconti, testi teatrali e saggi. La sua narrativa esplora le linee di confine fra l'umano e l'animale, le relazioni famigliari, la violenza e il lutto, attraverso ambientazioni distopiche, come in Sirene (Einaudi, 2007; Marsilio, 2017), finalista al Premio Bergamo 2008, o di soglia fra il reale e l'onirico, come in La ragazza selvaggia (Marsilio, 2016) finalista al Premio Campiello, e La metà di bosco (Marsilio, 2018).

12 Caterina Serra è nata a Padova nel 1969 e vive a Venezia, sua città d'adozione dopo molti anni passati in Inghilterra. È autrice di Tilt (Einaudi, 2009), una raccolta di reportages sulle vite segregate di persone affette da MSC (Multiple Chemical Sensitivity), e del romanzo Padreterno (Einaudi, 2015), in cui racconta di una relazione d'amore e di abuso attraverso le parole di un uomo violento. Scrive anche per il cinema, per cui ha curato la sceneggiatura di Piccola Patria (2013) ed Effetto Domino (2019) per la regia di Alessandro Rossetto, e dei documentari Parla con lui (regia di Elisabetta Francia, 2010) e Napoli Piazza Municipio (regia di Bruno Oliviero, 2008). Lavora come editor per diverse case editrici e collabora con L'Espresso e Il Manifesto, e porta avanti progetti di fotografia e danza contemporanea.

Nadia Terranova è nata a Messina nel 1978 e vive a Roma. È autrice dei romanzi Gli anni al contrario (Einaudi, 2015), vincitore fra gli altri del Bagutta Opera Prima e del Premio Brancati, e Addio fantasmi (Einaudi, 2018), finalista al Premio Strega 2019 e vincitore di numerosi altri premi. Ha pubblicato diversi libri per ragazzi, fra cui Caro diario ti scrivo... (Sonda, 2011), Bruno il bambino che imparò a volare (Orecchio Acerbo, 2012) e Omero è stato qui (Bompiani, 2019), e il saggio Un'idea di infanzia. Libri, bambini e altra letteratura (Italo Svevo Editore, 2019). Collabora con diverse testate tra cui Repubblica, Internazionale e Il Foglio, è docente alla Scuola del libro di Roma e collabora con Radio Due Rai. I romanzi, di stampo realista, si concentrano sui rapporti famigliari e sul rapporto fra genitori e figli, in particolare sul tema dell'assenza.

\section{Autorialità}

Elsa Morante si definiva 'scrittore', usando per sé il maschile, inteso come neutro universale, a cui contrapponeva il rifiutato 'scrittrice', visto come rappresentativo di una parzialità ${ }^{13}$. Anna Maria Ortese, in Corpo celeste, metteva in luce le tensioni e le contraddizioni intrinseche alla posizione occupata dalle scrittrici, definendosi «uno scrittore-donna, una bestia che parla, dunque ${ }^{14}$. A partire dalle posizioni di Morante e 
Ortese, ho domandato alle scrittrici se, secondo loro, c'è più spazio oggi per definirsi scrittrice rispetto alle generazioni precedenti, e se loro stesse si definiscono scrittrice.

Tutte le autrici intervistate utilizzano, per definirsi, il termine scrittrice e ritengono che sia più semplice utilizzare il femminile oggi, ma mettono in luce le tensioni che permangono intorno all'uso del femminile. Janeczek collega una più diffusa assunzione del termine scrittrice a una crescente consapevolezza collettiva del differenziale di status fra scrittori e scrittrici e della necessità di un riconoscimento reciproco:

Sì, io mi definisco scrittrice, io come altre cominciamo a rivendicare il fatto che possiamo essere insieme donne e produttrici di qualcosa che sia letterariamente altrettanto strutturato dell'opera di un collega. Io credo che da parte delle scrittrici, anche da parte di generazioni diverse, negli ultimi tempi ci sia stato un rendersi conto che le condizioni di ascolto e di riconoscimento non fossero pari a quelle dei colleghi e quindi c'è stato almeno un tentativo di mutuo riconoscimento. Anche delle scrittrici che tutto sommato non si sentono tanto toccate da tutto questo percepiscono la difficoltà di veder riconosciuto il proprio status e forse sottoscrivono anche l'idea che questo sia un problema comune, e che non basti dimostrare ognuna di essere brava. (HJ)

16 Serra rivendica per sé il termine scrittrice, e mette in luce come l'autorevolezza sia storicamente associata al maschile e come questo potenzialmente perduri anche oggi, allo stesso tempo aspirando a un superamento del binarismo di genere:

Non mi definisco scrittore, però capisco perché ci sia tutt'oggi qualcuna che decide di dire 'sono scrittore': dà conto di una storia e di una cultura che riconosce nel maschile il saper fare, il saper essere, rispetto a chi però? Il dato normativo, il maschile come potenza, come definizione in rapporto a cui dovrei stare, già Lonzi lo rifiutava. Oggi, preferirei parlare di no gender, di nessuna distinzione di genere, dove il genere è stato ed è funzionale al sistema patriarcale ancora dominante. Vorrei che ogni identità smettesse di avere un ruolo, ogni esistenza valesse alla pari, senza logiche di oppressione, colonizzazione. Vorrei poter finire ogni parola con una $\mathrm{U}$ o un asterisco! (CS)

17 Terranova osserva che, sotto il piano linguistico, la questione di fondo sia quella della parità di percezione e trattamento fra scrittori e scrittrici, e cerca invece una continuità rispetto alla scelta di Morante, interpretandola come orientata allo stesso fondamentale desiderio di parità in un contesto di disparità:

Io credo che oggi si dica scrittrice e io preferisco dire scrittrice, perché credo che forse l'affermazione della parola al femminile possa pian piano mettersi sullo stesso piano di quella maschile e cercare di recuperare il terreno perduto. Penso che il passo indietro che faceva Elsa Morante andasse comunque nella stessa direzione in cui oggi si va cercando di affermare la parola scrittrice al femminile, nel senso che l'obiettivo è comune, le strade per raggiungerlo sono diverse. L'obiettivo comune è quello di considerare scrittrici e scrittori su unico piano. È una lotta difficilissima. (NT)

18 Al tema del genere e dell'autorialità si lega quindi chiaramente il problema dell'autorevolezza ovvero dello status, del riconoscimento di valore. La prospettiva di Pugno, per esempio, è politicamente improntata alla messa in luce delle posizioni di potere che si esplicitano negli usi linguistici, per cui il termine 'scrittrice' è percepito $\mathrm{e}$ usato consapevolmente in senso militante, accanto ad altri termini che denotano posizioni di autorità.

I termini e le parole che usiamo sono storicamente determinati. Io inizio scrivendo poesie e continuo a scrivere poesie, quindi la mia prima definizione è poeta o poetessa. Di mio sarei abbastanza indifferente all'essere definita poeta o poetessa, però in realtà facendo il lavoro che faccio - dirigo l'Istituto di Cultura di Madrid - 
mi sono posta per esempio la questione del se farmi chiamare direttore o direttrice, che sono ovviamente termini molto connotati, quasi più che scrittore e scrittrice. La mia posizione da un certo punto di vista è abbastanza pragmatica. Noi siamo abituati al suono e all'uso di determinati termini, pensiamo a studentessa o professoressa che oggi ci sembrano assolutamente normali ma ai primi del Novecento sembravano degli abomini linguistici perché era nuovo il fatto che le donne accedessero a determinati ruoli. Così come si è dibattuto su infermiera sì, ingegnera no. Queste parole suonano stonate perché il nostro orecchio si è formato in un determinato modo. Detto questo, io ho fatto una consapevole scelta di usarle, anche quando magari il loro suono mi sembri stridente, semplicemente perché lì ove è esistita una funzione di potere nella storia è sempre esistita anche la parola: diciamo regina, principessa e sacerdotessa. (LP)

Cilento adotta per sé senza esitazioni la definizione di scrittrice, e mette l'accento su quello che secondo lei è un grande cambiamento avvenuto rispetto alla generazione di Morante e Ortese, ovvero la possibilità di affermarsi come scrittrice indipendentemente dal supporto autorevole di un uomo, ma riconosce a sua volta come la percezione di prestigio graviti ancora intorno al maschile.

Sicuramente è più facile oggi definirsi scrittrice rispetto ai tempi di Elsa Morante, dove la maggior parte delle scrittrici italiane dovevano avere qualcuno accanto. Per paradosso Morante è in assoluto la più grande anche per aver avuto accanto per tanti anni Alberto Moravia, che rendeva la cosa in qualche modo socialmente più accettabile. E lo stesso è valso per Anna Banti, che è un'altra grandissima, però che era riuscita ad essere rilevante anche perché moglie di Roberto Longhi. Ed è vero anche che per esempio per Anna Maria Ortese il fatto che non ci fosse un uomo accanto per lei ha pesato moltissimo, per la sua storia editoriale e per le sue vicende. Certamente non siamo più negli anni Cinquanta o Sessanta, e dirsi scrittrici oggi dovrebbe non essere un problema. Tuttavia ci sono anche tante scrittrici o poetesse viventi che insistono per farsi chiamare ancora poeta o scrittore, perché in qualche modo dobbiamo ancora conquistarci un completo rispetto, un totale riconoscimento. (AC)

Dalle conversazioni emerge una radicata consapevolezza della politicità del linguaggio, e una scelta altrettanto consapevole di rivendicazione di status attraverso il femminile. Rispetto al passato, il posizionamento soggettivo delle scrittrici è sicuramente rinforzato, essendo cresciuto lo spazio di azione per le donne, mentre non sembra essere cresciuto il riconoscimento, cosa che si riflette nella percezione, da parte per esempio di Serra e Cilento, che molte donne possano ancora optare per il maschile in cerca di autorevolezza, mentre Janeczek, Pugno e Terranova enfatizzano piuttosto la dimensione collettiva della scelta del femminile 'scrittrice'. Se tutte le scrittrici intervistate usano il femminile, la scelta ha ancora carattere consapevolmente militante e si inserisce in un quadro in cui un'oscillazione fra pratiche diverse sembra essere ancora possibile.

\section{Canone e genealogie letterarie}

21 La seconda area tematica affrontata nelle interviste riguarda la dimensione interpersonale del legame con altre scrittrici, del passato e del presente. Ho domandato alle scrittrici se, per loro, esiste una genealogia di scritture di donne e, se sì, se ricopre un ruolo rilevante per il loro lavoro di scrittrici oggi. Dalle interviste emerge che, mentre esiste una conoscenza e un apprezzamento delle opere delle scrittrici, queste non risultano però svolgere una funzione a livello di genealogie per quanto riguarda il 
genere. Nessuna delle autrici, ad eccezione di Serra, opera una distinzione fra scrittori e scrittrici dal punto di vista della lettura, considerando entrambi come parimenti importanti e fondanti per il proprio mestiere e rivendicando tale parità indifferenziata come un valore. Non emerge, in altri termini, un repertorio tematico o discorsivo sentito come femminile, legato a una genealogia di scrittrici, ma al contrario una tensione al superamento di qualunque specificità sessuata verso un mondo letterario pluralistico. D'altro canto, diverse autrici spostano l'attenzione sul problema della marginalizzazione delle scrittrici del passato nel canone letterario e sulla necessità di farsi carico di un'operazione di recupero e valorizzazione.

Janeczek sottolinea la necessità che i testi delle scrittrici siano in primo luogo disponibili e accessibili, e in secondo luogo auspica che si possa raggiungere un riconoscimento del loro valore che prescinda dal gusto personale del singolo critico (che è uno degli argomenti più spesso usati in difesa di selezioni escludenti, e che non ha un corrispettivo quando si tratta di assegnare uno spazio a scrittori).

Penso che sia importante fare attenzione che le scrittrici canonizzabili o canonizzate di fatto rimangano nel canone, perché altrimenti non si riesce neanche a scoprirle. Sarebbe bello che la lettura e l'apprezzamento delle opere delle scrittrici più importanti fosse un'opzione sia per le donne che per gli uomini, però per fare questo bisogna prima farle conoscere. Io credo che la letteratura sia anche una cosa in cui non necessariamente tutti amano tutti, visto che nella letteratura portiamo la complessità della nostra configurazione e tutto ciò che ci determina $\mathrm{e}$ che sfugge anche alle determinazioni - donne, uomini, origini, orientamento sessuale, storia, classe, psicopatologie o nevrosi di famiglia, insomma tutto quello che siamo lo portiamo con noi. Ma sono convinta che esista un livello di qualità e di importanza che possa essere riconosciuto a prescindere da quanto quella scrittrice parli a ciascuna di noi. Ad esempio io non amo particolarmente Marguerite Duras, però non mi sogno di dire che Marguerite Duras non sia stata una grande scrittrice, no? È un discorso di obiettivo comune e condivisibile, culturale e politico. (HJ)

Questa la selezione personale di Janeczek, la quale sostiene chiaramente che, a livello di lettura, non c'è per lei una distinzione fra scrittori e scrittrici, e che questa pratica dovrebbe essere un'opportunità per tutti:

Ci sono alcune scrittrici e poetesse che ho amato molto. Quella da me più amata è Ingeborg Bachmann, sia poetessa che narratrice che persona che riflette sulla letteratura e non solo. Ho un buonissimo rapporto con Virginia Woolf. Amo molto, non credo di somigliarle ma amo molto Katherine Mansfield. Non tifo in particolar modo per nessuna delle grandi scrittrici del '900 italiano perché mi piacciono tutte. Ho avuto però la fortuna di essere molto sostenuta e adottata da Lalla Romano, che è una scrittrice che oggi è un po' uscita dal focus, così come altre che stanno sbiadendo, e non mi sembra una buona cosa, per esempio Fabrizia Ramondino. Il bello della letteratura o in genere dell'arte è proprio la diversità, per cui per me non è un problema aver amato dei libri scritti da uomini. Io sono abbastanza allergica ai discorsi un po' prescrittivi, mentre sono fortissimamente favorevole al fatto che ci sia un obiettivo comune, che è quello di cercare tutti di far sì che tutte le scrittrici di un certo rilievo siano debitamente considerate, e che chiunque voglia mettere naso nel campo della letteratura abbia una possibilità di trovare quella che più gli piace. $(\mathrm{HJ})$

Parlando del rapporto con le scrittrici del passato, Serra racconta di spendersi personalmente, attraverso la propria attività culturale, per promuovere l'esistenza e la diffusione dei testi delle scrittrici, combattendo contro quello che vede come un oblio sistematico: «Lavoro molto sul restituire spazio, sul restituire memoria alle donne che hanno scritto, che come scrittrici nessuno conosce vede ha letto. Hanno deciso che non 
c'erano e nella narrazione maschile sono state fatte fuori». Serra è anche l'unica, fra le intervistate, ad affermare l'importanza per sé di una genealogia di scrittrici a partire da un diverso attraversamento del mondo da parte di uomini e donne, ma chiarisce anche come tale diversità sia da intendersi come un costrutto storico-culturale e non un dato essenzialistico:

Sento enormemente una genealogia di scrittrici, ma non perché hanno scritto come donne. Questa fasulla discussione polemica sullo scrivere al femminile o al maschile la contesto radicalmente, sono convinta che non ci sia uno stile delle donne o degli uomini, però sono altrettanto convinta che ci sia un attraversamento dell'esperienza della vita dissimile, che a partire dai corpi, dal desiderio e dalla partecipazione nel mondo, la realtà e la sua percezione cambino. Non geneticamente, identitariamente, ma perché una certa storia e cultura, una certa biopolitica, ha costruito un modo di guardare il mondo. [...]

Molti dei miei modelli di riferimento sono donne, cito spesso più donne scrittrici filosofe e pensatrici femministe. È lì che trovo consapevolezza, rivoluzione dello sguardo, spostamento. Virginia Woolf per prima. Ma poi, Lonzi, Sontag, Haraway, Paley, fino a Despentes. Poi, non faccio che citare Preciado, a proposito di: l'identità non esiste. (CS)

Pugno parla invece chiaramente di una pratica di lettura mista e indifferenziata:

Ho sempre letto scrittrici e scrittori allo stesso modo. Però no, non ho avuto una storia di ricerca di autrici donne in particolare. Ovviamente ho letto scrittrici, Virginia Woolf, Adrienne Rich... Però non le ho messe in una genealogia legata al fatto di essere scrittrici, è forse qualcosa di più ampio. Noi diventiamo scrittrici e scrittori nell'amore per la letteratura che è stata scritta prima di noi. Senza quell'amore non scatta in te la scintilla. Però per me questo è venuto allo stesso modo da uomini e donne. (LP)

In questo senso, la differenza fra uomini e donne, che pure si pone in modo così forte a livello sociale, viene considerata da Pugno come superata o superabile nella scrittura e nella lettura di testi letterari:

Io credo, a un livello molto profondo, di continuare a considerare l'asimmetria in cui continuamente mi imbatto, il fatto che il mondo sia organizzato molto diversamente per un uomo o per una donna, tuttora, non so come dire, come una sorta di sovrastruttura. Nel mio essere profondo, gli uomini e le donne sono talmente pari, sono talmente la stessa cosa nelle loro diversità che tutto questo è quasi inconcepibile. (LP)

27 È sul piano politico e culturale che invece Pugno pone la questione della differenza fra uomini e donne come predominante in tutti i campi artistici, ed è su questo piano che agisce in quanto direttrice dell'Istituto di Cultura di Madrid per cambiare la situazione. Pugno sottolinea come una forma di riconoscimento e valorizzazione reciproche fra donne sia fondamentale a questo scopo, insieme a un'azione che interrompa i pregiudizi e i condizionamenti interiorizzati che portano alla riproduzione delle gerarchie di genere:

Io organizzo eventi, festival e manifestazioni in quasi tutti i campi: mostre, cinema, arte, letteratura, design, cucina. Ciò che la realtà in quasi qualsiasi campo mi restituisce è una proporzione di uomini e donne rispettivamente del $70 \%-30 \%$, magari del 60\%-40\% in alcuni settori, o dell' $80 \%-20 \%$ in altri. Le registe sono mosche bianche; le artiste sono già più numerose, ma sono comunque meno. [...] Dove tu vedi che ci sono delle donne in posizioni di potere, automaticamente aumenta il numero di donne, anche semplicemente perché una donna è abituata a percepire le altre donne. Perché ci sono anche persone solitamente democratiche nell'atteggiamento mentale che però non si rendono conto di questa disparità strutturale. [...] Il mondo letterario, il mondo artistico, è in linea generale uno dei 
più avanzati. Paradossalmente per questa ragione moltissimi condizionamenti passano a livello inconscio, non ne siamo tuttora consapevoli nella nostra stessa pratica, anche perché ci vengono rimandati costantemente. (LP)

\section{fondamentale sulla sua scrittura. Sebbene in nessuno dei due casi la rilevanza sia} collegata al genere - Ortese è citata per la rappresentazione dello stesso contesto geografico e sociale napoletano di Cilento, Banti per il ruolo della pittura - Cilento si sofferma anche sul fatto che «entrambe sono delle donne che hanno dovuto terribilmente lottare per poter scrivere». Cilento passa poi a mettere in rilievo la differenza nella formazione letteraria di uomini e donne, che è spesso orientata alla lettura esclusiva o quasi esclusiva di scrittori da parte degli uomini, mentre per Cilento, come per le altre autrici, è stata sempre mista:

Gli uomini non leggono le donne. E questo riguarda sia la lettura dei classici, appunto di scrittrici del passato, e riguarda ancora di più il presente. Non le leggono e non si spendono per loro. Per me sono ugualmente importanti autori che hanno costituito la base della mia formazione, da Stevenson a Bulgakov, da Hoffman a tutto il coté fantastico a Cechov - non posso pensare di scrivere senza far riferimento diretto a Tolstoj, a Flaubert, Maupassant... Così come c'è tutta una costellazione di donne, dalle sorelle Brontë, Marguerite Yourcenar, fino ad arrivare appunto alle nostre scrittrici. (AC)

Anche Terranova parla, più che di genealogie, della libertà di leggere tutto, contrapposta da un lato alle scelte di lettura limitate che osserva da parte di altri scrittori, e dall'altro all'enfasi esclusiva su genealogie femminili:

Gli scrittori non citano mai o quasi mai scrittrici se non con piccole eccezioni. Si vede che quando lo fanno è per inserire una quota. Noi siamo molto più libere. A volte sento un po' il peso di alcune scrittrici più ideologizzate, magari nel dialogo, non nella scrittura, che citano forzatamente solo scrittrici, e anche quello è un po' strano perché nella tua vita di lettore tu incontri i libri, e poi successivamente incontri i generi. (NT)

La libertà di lettura si riflette nell'indistinzione fra scrittori e scrittrici citati per quanto riguarda la propria formazione. Tuttavia, senza che venga messo a tema, Terranova è anche, fra le autrici intervistate, quella che arriva più vicina a una prospettiva di tipo genealogico, attribuendo un ruolo di scoperta e iniziazione alle opere di Natalia Ginzburg e Alba de Céspedes che ha a che fare specificamente con la posizione sociale delle donne e l'articolazione di tale condizione nel romanzo:

Fra le scrittrici, quella a cui sono più debitrice è Natalia Ginzburg nella sua completezza. Alba de Céspedes è un'altra che amo moltissimo, perché Quaderno Proibito come Lessico famigliare mi hanno insegnato una cosa importante, cioè che tu per scrivere un grande romanzo potevi farlo stando nel chiuso di una casa e di una famiglia. Io ho sempre sentito che la mia narrativa - prima ancora che diventasse la mia narrativa, la mia immaginazione - si era nutrita di tutto quello che era successo nelle mura di casa. Libri come Quaderno proibito e Lessico familiare, ma anche Caro Michele, mi hanno detto questo: che io potevo prendere il materiale che avevo e con quel poco farci romanzi interi. [...] Pensa a Quaderno Proibito in fondo, è una storia di nulla, è la storia di una donna che nella scrittura di un quaderno trova il suo giardino segreto e che piano piano lo trasferisce poi nel rapporto con il suo capo di lavoro, in quei pomeriggi che più che un adulterio sono un'affermazione di libertà, come dire, posso essere un altro tipo di donna rispetto alle regole che la società mi impone. Sono dei movimenti piccolissimi, eppure è un romanzo sconfinato. (NT) 


\section{Rapporto con la critica, il pubblico, l'editoria}

31 Nel breve brano Il mio mestiere, Natalia Ginzburg scrive: «Desideravo terribilmente di scrivere come un uomo, avevo orrore che si capisse che ero una donna dalle cose che scrivevo. Facevo quasi sempre personaggi uomini, perché fossero il più possibile lontani o distaccati da me» ${ }^{15}$. Secondo Raffaella Silvestri, «la libertà di scrivere di sentimenti senza sembrare sentimentali, toccare le emozioni senza essere chiamati emotivi: una prerogativa ancora maschile, con cui la scrittura femminile deve fare $\mathrm{i}$ conti $»^{16}$. A partire da queste considerazioni, la terza area tematica delle interviste verte sul rapporto con la ricezione. Ho chiesto alle scrittrici (1) se c'è una differenza di status fra scrittori e scrittrici oggi in Italia; (2) se avvertono una pressione da parte del pubblico, della critica, dell'editoria o della società in senso lato sulla propria scrittura, legata al fatto di essere donne; (3) se riscontrano una differenza nella ricezione delle opere delle scrittrici rispetto a quelle degli scrittori.

32 All'interno della varietà delle risposte e degli approcci, che vanno dal sociologico al filosofico al personale, emerge come dato condiviso l'idea che gli stereotipi di genere riguardino le lenti applicate nel momento della lettura e dell'interpretazione molto più che la realtà stessa delle opere. Janeczek riscontra per esempio come ci siano precise aspettative di genere che orientano la lettura dei testi a seconda che siano scritti da un uomo o una donna, e come il diverso orientamento ermeneutico incida sulla percezione di letterarietà:

La griglia mentale dice che le donne sono più emotive, più sensibili, più a contatto coi propri sentimenti, stanno in genere in una dimensione privata, quindi parlano di cose intime, di rapporti e relazioni famigliari eccetera. In più è passata l'idea che parlare di relazioni tra persone normali, nelle famiglie, sia femminile, o perlomeno quando sono descritte da una donna sono femminili, quando sono descritte da un uomo no. Kent Haruf che scrive di un microcosmo in cui succede poco e che ha avuto un grandissimo successo è bravo perché sa scrivere la normalità; se questo fosse venuto fuori dalla penna di una scrittrice sarebbe stato più difficile considerarlo parimenti come letteratura. (HJ)

Janeczek porta quindi un esempio tratto dalla propria esperienza personale riguardante la ricezione de Le Rondini di Montecassino, ambientato durante la seconda guerra mondiale. Janeczek riporta che in diverse occasioni le è stato domandato da parte dei critici come fosse per una donna scrivere di guerra, tema percepito come di appannaggio maschile, a cui lei replica:

Per me la guerra e l'esito della guerra sono delle condizioni sine qua non della mia esistenza. Lo sento come uno scenario che mi appartiene, in modo diverso ma credo non meno stringente di qualche collega che ha un fascino culturale tipico del suo essere cresciuto come maschio verso l'ultima guerra o altre guerre in generale. Perché in realtà nella mia generazione e da lì in avanti anche gli scrittori non hanno certamente vissuto la guerra, quindi non ne sanno molto di più per nascita rispetto a me. Loro però possono lavorare su una tematica che ha a che fare con il loro ruolo nei secoli che non muta nel momento in cui le guerre in Occidente non si fanno più (per il momento). (HJ)

Le aspettative di genere, radicate negli stereotipi sociali, si riproducono, osserva ancora Janeczek, in una dinamica circolare, di cui fanno parte l'attitudine della critica e l'industria editoriale:

In genere la comunicazione alla fetta delle lettrici, che pure soprattutto in Italia è quella che tiene in piedi tutta la macchina editoriale, è fatta con delle copertine 
molto genderizzate. Ci sono fanciulle pensose in copertina, belle immagini femminili, tazzine di tè o di caffè... Caratteristiche che di per sé non vogliono dire niente rispetto alla qualità del lavoro che è stato fatto su questi temi, perché i temi delle relazioni sentimentali e famigliari sono quelli al centro di buona parte del romanzo cosiddetto borghese, quindi del romanzo come noi l'abbiamo in mente tout court. Quando esce dalla penna di una scrittrice, questa serie di temi in genere viene presentata come un qualcosa che si rivolge direttamente da donna a donna, con una complicità sul fatto che i contenuti siano accessibili, e sulla base di un supposto bisogno delle lettrici di avere un'immediata identificazione, di essere anche loro interessate al godimento emotivo e non tanto all'approccio riflessivo. (HJ)

La stessa immagine di circolo vizioso è utilizzata da Pugno, che torna sul problema del diverso tipo di aspettativa che si applica ai testi scritti da donne e da uomini:

In realtà se noi pensiamo a grandi casi di amori letterari del passato sono praticamente tutti scritti da uomini. Perché le donne non scrivevano, ma anche perché l'amore e i sentimenti non sono appannaggio esclusivo delle donne. È chiaro che diventa una specie di uroboro, di serpente che si morde la coda, cioè all'interno di una determinata visione e percezione sociale io uomo non posso comprarmi non dico Bridget Jones, ma il romanzo di Donatella Di Pietrantonio, perché non leggo storie scritte da donne. E le lettrici sono indubbiamente molte più dei lettori, e leggono tutt'e due, però i livelli più alti, i vertici, i guardiani della soglia continuano a essere quasi tutti uomini. (LP)

Serra sviluppa una riflessione sul filo che tiene insieme emozioni, donne e letteratura minore, mettendolo in relazione alla svalutazione della dimensione del corpo nel discorso patriarcale, di contro al quale Serra cerca da un lato di spezzare il legame deterministico ed esclusivo fra scrittura delle donne e dimensione emotiva/personale/ corporea, e dall'altro di riabilitare quella stessa dimensione.

Ci hanno raccontato che le donne hanno scritto dell'emozione, dell'amore, dei sentimenti, di una certa interiorità. È una narrazione imposta, una misdirection, una deviazione dello sguardo e dell'attenzione. Hanno deciso, per sottolineare che le scrittrici facevano un lavoro minore, che gli affetti, le emozioni, l'amore, ciò che è tratto dalla esperienza personale, è minore. Ma questa è una concezione patriarcale, è la narrazione del mondo e della realtà raccontata dagli uomini, che hanno escluso dal potere, dalla rappresentazione pubblica di sé, la parte appunto più umana della vita, la morte, gli affetti, l'amore, la nascita, il corpo in generale. Si genera così l'idea che sia minore la scrittura che riguarda il corpo, come se l'intellettualità e il sapere non avessero a che fare con la corporeità. Come se non fossimo corpo, come se mente e corpo non facessero parte dello stesso sistema percettivo, cognitivo. Bisognerebbe rileggere Spinoza, Deleuze, Braidotti, anche. Il corpo, i corpi: tutto quello che siamo. (CS)

Cilento riscontra una maggiore incidenza degli stereotipi man mano che si va verso prodotti più esplicitamente commerciali, e introduce un tema molto sentito dalle autrici, quello del riconoscimento della "letterarietà alta" ${ }^{17}$. Come Serra parla di una gerarchia fra sfera corporea femminile e dimensione intellettuale maschile, così Janeczek sottolinea il problema che le aspettative genderizzate comportano per il riconoscimento del valore letterario dei testi scritti da donne:

Il twist più spinoso è che si fa in genere fatica a pensare alla scrittura delle donne come qualcosa che possa raggiungere dei risultati di grandissima letterarietà anche quando il lavoro è effettivamente su questi temi. Esiste una letteratura che apparentemente non ha bisogno di grandi costruzioni e che però è assolutamente eccellente, no? E questo a una donna si ha chiaramente più difficoltà a riconoscerlo. Si fa difficoltà a riconoscere a delle scrittrici, forse in generale a delle artiste, il fatto 
che possano avere un approccio anche riflessivo, cioè che siano veramente tanto quanto i colleghi consapevoli del loro percorso, che possano essere delle intellettuali, che abbiano delle idee sofisticate, perché anche lì c'è questa specie di pregiudizio del cuore, della sensibilità, dell'immediatezza. (HJ) culturale e politico in generale:

Il solo fatto che non ci sia mai stata una direttrice di giornale dei giornali più venduti in Italia è gravissimo, ma è ancora più grave il fatto che se accadesse si direbbe 'al femminile' e si porrebbe l'attenzione sul fatto che c'è una donna, non sulle sue competenze o su quello che fa. Questo se lo guardiamo dall'esterno è proprio una segregazione incredibile, perché c'è quello che viene chiamato il soffitto di cristallo che non si riesce a sfondare. Tu non lo vedi, non c'è di fatto un veto sul fatto che le donne possano essere Presidente della Repubblica o Direttore del Corriere della Sera, ma di fatto accade. E con i premi letterari accade la stessa cosa. Pensa che cosa sarebbe il nostro paese se per quindici anni il Premio Strega l'avesse vinto una donna: sarebbe stato considerato un premio per ricamatrici. Cosa che invece non avviene per gli uomini, non si pensa che il premio Strega è una cosa per maschietti che giocano a football perché l'hanno vinto i maschi. (NT)

Terranova poi menziona un altro punto rilevante, ovvero ipotizza che il pregiudizio dei lettori uomini sia più forte nei confronti delle scrittrici italiane rispetto a quelle straniere, osservazione che chiama in causa il ruolo delle donne all'interno della nazione:

Gli uomini leggono le donne molto meno di quanto leggano gli altri uomini, e con moltissimi pregiudizi, soprattutto sulle connazionali. Gli uomini se devono leggere una donna leggono Il cardellino di Donna Tartt, perché è una scrittrice che sta in un universo diverso dal loro. (NT)

Dall'altro lato, Janeczek ipotizza che parte del proprio successo sia dovuto anche al fatto di non essere percepita primariamente come donna, a causa di altri fattori 
biografici - inclusa l'origine straniera - e tematici che vanno a comporre il suo profilo autoriale:

Io sono talmente una bestia strana per tanti aspetti, cioè per il multilinguismo, per la storia strana che ho alle spalle, perché il mio cognome non è italiano, che ho avuto un trattamento diverso dalle mie colleghe, perché non apparivo mai solo come una donna. È brutto a dirsi però va detto anche così, mi portavo dietro spezzoni di storia di interesse anche del lettore uomo. (HJ)

Pugno ritiene di riuscire a intercettare un pubblico misto anche grazie al fatto di scrivere libri "letterari", e di essere per questo percepita meno secondo le stereotipie di genere, soprattutto con il romanzo Sirene, ancora una volta segnalando come l'autorevolezza passi dalla possibilità di superare tutto ciò che è percepito come femminile e così rimarcando la posizione assiologicamente inferiore del femminile stesso:

Non mi è mai sembrato che i miei libri fossero letti esclusivamente da donne. I miei sono libri letterari. E sono letti anche da uomini. Sirene in particolare. Il mio lettore non è né uomo né donna, è tutti e due, o insomma io spero che lo sia. Anche perché nell'idea di parlare solo alle donne o di parlare solo agli uomini si riannida la stessa asimmetria. [...]

Una volta Alessandra Sarchi mi disse: «Tu non sei percepita come una scrittrice, sei percepita come uno scrittore». Questo perché non ho quasi mai, nelle mie scritture, praticato temi tradizionalmente - tra tutte le possibili virgolette - 'femminili': casa, famiglia, coppia, figli, eccetera. I miei libri tendenzialmente parlano di altro. Ci sono storie d'amore indubbiamente, però il contesto, il modo, la cornice in cui le scrivo non è quella della scrittura tradizionalmente identificata come femminile. (LP)

Anche per Terranova una delle possibili ragioni del successo de Gli anni al contrario presso un pubblico misto è l'attraversamento di temi politici e quindi non stereotipicamente femminili:

Ho avuto molte lettrici però devo dire che ho avuto anche molti lettori. Anche perché Gli anni al contrario è stato percepito come un libro che comunque attraversava lo Zeitgeist degli anni Settanta. E quindi ho avuto molti lettori uomini. Spero che mi seguiranno anche in Addio fantasmi che invece ha questa presenza femminile è molto forte. (NT)

Di fronte a tali e tanti condizionamenti, incomprensioni e negoziazioni, tuttavia, il dato che accomuna tutte le autrici intervistate è l'affermazione di una piena libertà personale nella propria scrittura. Serra per esempio confronta la propria attività di sceneggiatrice per il cinema, che trova essere pesantemente condizionata e silenziata dalla dominanza maschile del settore, con la propria attività di scrittrice, che le permette invece un rapporto più libero: «nella scrittura sono io da sola e poi ho la reazione dei lettori e della critica, è più solitario, mentre la mia scrittura per il cinema è deviatissima dalla relazione col maschile». Questa libertà, che per Serra è anche rifiuto di sottostare al lavoro promozionale richiesto dal sistema editoriale, è rivendicata anche laddove ritiene possa portare a un mancato apprezzamento:

Io non credo che abbiano letto Padreterno, i cosiddetti critici, ma ci sono ancora? [...] O forse semplicemente non piace come scrivo, che va benissimo. Non credo che scriverei mai in un altro modo per compiacere qualcuno, tanti, un movimento, un'epoca, uno spirito del tempo. Cerco di raccontare una storia, sì, ma cerco anche il modo per raccontarla fino allo sfinimento, cerco la voce che la racconti, la cerco anche per anni. (CS) 
Di piena e rivendicata libertà parla Cilento: «Sono totalmente libera. Poi c’è il mercato, la critica... Ho avuto pesanti difficoltà e le ho a tutt'oggi, però nella creazione io sono potente, posso fare qualunque cosa»; si esprime in termini simili Pugno, ponendo l'accento sull'indipendenza economica e psicologica come fattori che consentono la libertà nella scrittura:

Io mi sento libera di scrivere ciò che voglio, nella piena consapevolezza che ogni libertà si paga centimetro a centimetro, e non solo relativamente al femminile ma a qualsiasi altro tipo di libertà. Io mi sento libera anche perché faccio un'altra professione con cui mi mantengo. Mi sono sempre sentita libera di scegliere i miei temi, non ho volontariamente evitato temi che avevano a che fare con il femminile tradizionalmente inteso. In realtà ho sempre pensato e vissuto il femminile come qualcosa di molto diverso, più ampio, più forte. [...] Per cui no, non ho mai scritto pensando devo o non scrivere questo perché altrimenti sarò percepita come una scrittrice. Quello che mi è chiarissimo è che devi poterti permettere la tua scrittura, rispetto a te stessa, alla tua famiglia, al tuo lavoro e ai tuoi rapporti col mondo editoriale. (LP)

Infine, la libertà sentita da Terranova tocca la sfera più intima della liberazione da ogni condizionamento e tabù che si afferma nell'atto creativo, mentre le pressioni intervengono a scrittura avvenuta, nel momento della ricezione:

Io devo essere sincera nella mia scrittura mi sento completamente libera. Sulla pagina non solo non avverto nessuna pressione né familiare, né sociale, ma se avverto una pressione vado ostinatamente ad affrontarla e dove c'era un tabù, anche della mia storia familiare, io l'ho preso e l'ho spiattellato. La mia scrittura nasce dove c'è una coercizione a non dire. Quando parlo, quando scrivo, sono molto più libera di quando vivo. Nel riconoscimento della scrittura, sento le pressioni. Quando il libro è fatto, il racconto è finito e si parla del libro, avverto che c'è una pressione, avverto il giudizio sul fatto di scrivere di personaggi maschili o femminili. (NT)

\section{Rapporto con il femminismo}

Il quarto e ultimo tema affrontato nelle interviste verte sulla relazione con il femminismo, e specificamente su due aspetti, ovvero se le scrittrici si definiscono femministe e sostengono le lotte femministe, e, in secondo luogo, se c'è una relazione fra la propria scrittura e il femminismo, e, se sì, di che tipo. Come nel caso della parola 'scrittrice', tutte le autrici si identificano con i principi e le lotte femministe, sebbene con gradi diversi di vicinanza personale e coinvolgimento politico attivo. Serra è l'unica, fra le intervistate, a fare parte di un movimento femminista, quello di Non una di meno. Pugno sostiene un impegno attivamente femminista attraverso il proprio lavoro di direttrice dell'Istituto Italiano di Cultura di Madrid, mentre per le altre scrittrici il sostegno si esplica più sul piano dell'assenso culturale che tramite un'azione diretta. Nel caso di Terranova, emerge un'idea di femminismo come postura individuale, a cui non fa riscontro un movimento specifico nel presente, e un distanziamento netto e sentito da quello che l'autrice definisce «femminismo biologico», visto come superato e normativo:

Io mi definisco femminista, è molto dura perché credo che oggi più di ieri non ci sia un femminismo, ma ci siano tanti femminismi, e non ne ho uno in questo momento che mi piaccia del tutto. Non mi rispecchio in molte donne, anche scrittrici, che si definiscono femministe. [...] La cosa che per me è un grandissimo no a prescindere è tutto il femminismo che si fonda sulla biologia. Per me è intollerabile pensare che 
nel 2018 ci sono donne che si definiscono femministe e che legano l'essere donna al discorso del corpo, alla mistica della maternità, tutto quello che è la Grande Madre, se non sei madre non puoi capire, la donna che dà la vita... (NT)

Anche Janeczek parla di una dimensione individuale di femminismo, e, dal punto di vista teorico, abbraccia una lettura intersezionale del femminismo. Sia Pugno che Cilento poi sostengono con forza l'idea che il femminismo debba necessariamente coinvolgere gli uomini, a partire da una decostruzione della contrapposizione fra maschile e femminile. Nelle parole di Pugno:

Io mi definisco femminista. Il mondo così com'è, per quanto riguarda il rapporto uomo donna, non è qualcosa che mi sta bene, essere femminista è questo. [...] Equesto è un cambiamento che deve necessariamente coinvolgere anche gli uomini, inevitabilmente, perché gli uomini sono di tutti i tipi, così come le donne. Io non ho una visione idealizzata del femminile, per cui così come il femminile è più ampio e complesso di come ci è stato raccontato, lo è anche il maschile. (LP)

Per Cilento, il femminismo, che prima considerava non più necessario, si lega principalmente a un'opera di tipo educativo sulle generazioni più giovani affinché non si perdano le conquiste del passato:

Pensavo che il femminismo storico stesse morendo con le persone che l'avevano fatto perché non ci veniva comunicato, ma che i nostri diritti fossero ormai acquisiti. Strada facendo purtroppo ho imparato, andando nelle scuole e guardando le generazioni più giovani a cui ho insegnato, che invece i ragazzini e le ragazzine stavano perdendo qualunque contezza di quello che era stato fatto, mi trovavo in scuole dove si partoriva a 13 anni, dove si allattava in direzione, cioè vedevo le stesse cose che negli anni ' 50 e ' 60 aveva visto mia madre, che ha insegnato a scuola per tutta la vita al Sud. Si capisce allora quanto sia urgente riprendere queste battaglie. Con l'idea che sia necessario rifondare un concetto di femminismo che naturalmente deve tener conto del fatto che il mondo è cambiato, e che il femminismo non si fa senza gli uomini. È un problema proprio di educazione culturale, alla relazione e al dialogo. Considerata questa trasformazione di senso, sono senz'altro femminista. (AC)

51 Per quanto riguarda il rapporto fra femminismo e scrittura, questo è interpretato in modi diversi da ciascuna autrice, anche sulla base di come esperiscono la propria posizione sessuata e l'impegno politico femminista. Per esempio, Serra afferma la valenza intrinsecamente politica della propria scrittura:

La mia scrittura non si può spostare dallo sguardo politico, io scrivo pensando che le parole agiscano politicamente. Quando è entrato in gioco il femminismo, abbiamo finalmente cominciato a pensare che quello che siamo lo siamo nelle relazioni, pubbliche e private, non si può prescinderne. Il dato politico sta qui. Gli affetti sono una parola chiave del femminismo e hanno rilevanza politica. (CS)

Per Janeczek, il rapporto con una prospettiva femminista si esplica in modo indiretto sul piano dei temi, e in particolare nella rappresentazione di personaggi femminili che scardinano tabù e semplificazioni, inclusa quella di fungere da modello emancipazionista, che Janeczek percepisce come una tendenza controproducente:

Credo che la scrittura, la letteratura, l'arte possano benissimo essere dichiaratamente militanti su una cosa, e possano essere ottime se militanti. Oppure, il servizio che fa un certo tipo di discorso può essere più laterale. Io nei libri che hanno sviluppato delle tematiche collegate anche col femminile ho lavorato su delle zone coperte se non da tabù almeno da stereotipie molto forti. Queste idealizzazioni, quelle del rapporto madre figlia ad esempio in Lezioni di tenebra, fanno parte di una cultura che usa l'idealizzazione come un mezzo di controllo e di attribuzione a un ruolo beatificato ma comunque subalterno. Nell'ultimo romanzo 
[La ragazza con la leica] io non ho voluto in nessun modo creare una protagonista che corrisponde a quel tipo di richiesta tipica di questo momento in cui il femminismo è anche un contenuto che si vende, e quindi sono richiesti modelli di empowerment. [...] È ovvio che c'è un primo livello in cui va benissimo ed è necessario che ad esempio nei libri di testo delle scuole tu possa incontrare una figura femminile che fa la pilota, l'astronauta, la scienziata, e non la casalinga o la maestra. [...] Mi torna più problematica questa richiesta di narrazioni in qualche modo esemplari, se non addirittura agiografiche, perché creano una distanza incolmabile dalla lettrice. (HJ)

Pugno rinforza invece la dimensione di assoluta libertà in cui si muove nel momento della scrittura, e come questa libertà si origini nella convinzione profonda di una dimensione indifferenziata, in cui la sessuazione è una fra le tante coordinate identitarie:

Secondo me al livello della scrittura, che è un livello più profondo, il problema non esiste. Io sono, tra le tante cose che sono, una donna, ma nessuna di queste cose mi definisce unicamente. Non mi sento una scrittrice particolarmente italiana. Non mi sento necessariamente neanche troppo figlia del mio tempo. [...] E quindi nella somma di tutte queste identità, e non identità, per me veramente lo scrivere è un atto libero. Cioè un atto che un soggetto compie come soggetto intero, come soggetto libero, che è quello che io credo che le donne sostanzialmente siano. Poi certamente esci di casa e ti trovi in un mondo che è molto diverso. (LP)

Così Terranova, che ricollega la libertà di scrivere superando le coordinate identitarie, fra cui «l'accidente biologico» di nascere donna, a un obiettivo intrinsecamente femminista, secondo l'ottica per cui la differenza di genere stessa è un costrutto patriarcale:

Io credo che la libertà di essere chi si vuole essere, che è appunto quella che poi pratica e insegna il femminismo non biologico, sia molto simile a quello che si fa nella scrittura, cioè probabilmente è un canale di comunicazione aperto fra le due cose. Scrivo per un'assoluta libertà, e sono femminista perché credo nell'assoluta libertà di chi voglio essere e posso essere. (NT)

Infine, nelle interviste con Pugno, Terranova e Cilento ricorre un collegamento fra la propria formazione, il proprio sguardo femminista e la propria libertà nella scrittura. Tutt'e tre queste autrici raccontano un'esperienza di libertà nell'infanzia e di essere cresciute in contesti famigliari dove veniva comunicata una parità di genere fra uomini e donne, e di essersi poi scontrate con la disuguaglianza di genere e il ruolo restrittivo del femminile in un secondo momento o al di fuori della cerchia famigliare. Cilento per esempio spiega di essere cresciuta «studiando e vivendo in un mondo dove nulla mi era stato impedito [...] in quanto donna», e di aver poi toccato con mano discriminazioni e disuguaglianze intorno a sé, anche nelle generazioni più giovani. Il contrasto, vissuto con sofferenza, fra la propria formazione e la società è raccontato anche da Pugno e da Terranova:

E io ricordo veramente quand'ero bambina, appunto essendo io cresciuta in una casa atipica degli anni ' 70 , in cui non c'erano queste differenze, quanto per me fosse violento, fosse percepito come un'ingiustizia, il fatto invece che la scuola, la società, il mondo mi rimandassero la necessità in quanto donna di dover fare altre cose, dovermi adattare ad altri codici, o che determinati codici fossero necessariamente quelli che tu dovessi desiderare. (LP)

Per me, per come sono stata cresciuta, non aveva nessuna importanza essere maschio o femmina. Mi hanno insegnato che potevo fare quello che volevo, non c'erano dei ruoli che dovevo avere, perché ho avuto la fortuna di crescere in una famiglia che in quel momento storico era politicizzata, fine anni Settanta, quindi, come dire, il femminismo per me è una cosa naturale. A un certo punto, a venti, 
venticinque anni, entrando piano piano nel mondo degli adulti, ho capito che non era così scontato, che invece c'era della misoginia nel mondo e nei miei confronti, dovuta al fatto che io neanche me ne accorgevo però ero femmina. E allora ho fatto un tipo di percorso diverso. (NT)

\section{Conclusioni}

Dalle conversazioni con Cilento, Janeczek, Pugno, Serra e Terranova emerge un quadro variegato ma non polarizzato rispetto alla dimensione di genere nel campo letterario italiano contemporaneo. Il primo dato trasversale è la forte consapevolezza sociopolitica delle dinamiche di genere che attraversano il campo letterario da parte delle autrici, segnalando come queste dinamiche si rendano palesi a chi le subisce e negozia nella propria pratica artistica, culturale e politica. Rispetto alle riflessioni di autrici di generazioni precedenti come Natalia Ginzburg, Anna Maria Ortese ed Elsa Morante, le scrittrici contemporanee mostrano di essere in possesso di un linguaggio e di strumenti condivisi per interpretare la disparità di genere nel campo artistico, che è vista in piena continuità con la disparità di genere a livello sociale.

In seconda istanza, emerge un senso di riconoscimento e solidarietà reciproci, per cui la questione della discriminazione di genere, così come la strada verso il suo superamento, non sono vissute a titolo individuale ma come una situazione collettiva che coinvolge tutte le scrittrici. Anche per questo aspetto le interviste registrano un cambiamento rispetto all'atteggiamento prevalente nelle generazioni precedenti, spesso orientato a uno sforzo solitario di affermazione del proprio lavoro, analizzato da Fanning $^{18}$. Nelle riflessioni per esempio sui premi letterari, sui manuali scolastici, sui festival e in generale sul riconoscimento di valore, la solidarietà fra le autrici prevale marcatamente sulla rivalità e la tendenza a distinguersi individualmente. Allo stesso tempo, è radicata la visione secondo cui il superamento delle asimmetrie esistenti si può raggiungere solo attraverso il coinvolgimento degli uomini verso lo stesso obiettivo.

L'identità di genere è riconosciuta come uno dei fattori che influenzano la propria scrittura accanto a molti altri, e non è in alcun modo sentita come fattore principale o determinante. Ricorre quindi un'aspirazione a fuoruscire dagli stereotipi che ingabbiano ed essenzializzano il femminile, nella direzione di un superamento della differenza sessuale a partire spesso da un'esperienza di parità nell'infanzia. Tale aspirazione si lega a un senso forte della propria autorialità e a una rivendicazione della propria libertà creativa, a cui però non fa riscontro una parità di ascolto e riconoscimento nella società. Infine, i rapporti con il femminismo sono vissuti in modi diversi, che variano dall'adesione ideale a titolo individuale, all'impegno per la trasformazione delle pratiche culturali, alla partecipazione a movimenti e collettivi.

Complessivamente, le interviste fotografano uno scarto molto forte fra la consapevolezza e la libertà autoriali rivendicate dalle autrici, da un lato, e il loro riconoscimento di valore sul piano della ricezione. In altri termini, si pone una tensione fra la percezione del proprio lavoro da parte delle autrici, sentito come portatore di istanze letterariamente significative, e il riconoscimento di tale lavoro da parte della critica, del pubblico e dell'editoria, che tende a ignorarle o a ricondurle all'interno di stereotipi di genere che ne determinano una parzialità assiologicamente inferiore. Tale scarto fra produzione e ricezione si inserisce in un contesto in cui l'intero campo 
letterario, dai ruoli dirigenziali nell'industria editoriale, nei quotidiani e nelle riviste alle cattedre universitarie, è ancora dominato da uomini e riproduce, in forma di uroboro - per usare l'espressione di Pugno - l'assiologia valoriale fra maschile e femminile. Dando voce alle soggettività femminili nel campo letterario, la prospettiva di genere adottata nelle interviste consente quindi sia di esplicitare i rapporti di potere esistenti sulla base del genere, sia di restituire la complessità, la varietà e a tratti anche le tensioni inerenti alle negoziazioni e alle pratiche di resistenza messe in atto dalle autrici, individualmente e collettivamente.

\section{NOTE}

1. I nomi dopo le citazioni sono abbreviati rispettivamente in AC, HJ, LP, CS e NT.

2. Per quanto riguarda una prospettiva decostruttivista nello studio sociologico della cultura, nel cui ambito di ricerca si inserisce questo articolo, il punto di riferimento teorico e metodologico fondamentale è P. Bourdieu, La distinzione. Critica sociale del gusto, Bologna, Il Mulino, 2001 (1979). Per la critica femminista dell'universalismo dominante, fra $\mathrm{i}$ tanti riferimenti nel contesto italiano particolare importanza hanno i lavori di A. Cavarero, di cui si veda il saggio fondativo: Per una teoria della differenza sessuale, in Diotima, Il pensiero della differenza sessuale, Milano, La Tartaruga, 1987, pp. 43-79; J. Butler, Undoing Gender, New York, Routledge, 2004; R. Braidotti, Nomadic Subjects: Embodiment and Sexual Difference in Contemporary Feminist Theory, New York, Columbia University Press, 2011 (1994), soprattutto per la capacità di coniugare pensiero della differenza e anti-essenzialismo.

3. Monique Wittig, Il pensiero eterosessuale, Verona, Ombre corte, 2019 (1992).

4. Sul tema dell'autorialità in relazione al genere nel contesto della letteratura italiana, si vedano per esempio: U. Fanning, Italian Women's Autobiographical Writings in the Twentieth Century: Constructing Subjects, Madison, Fairleigh Dickinson University Press, 2017; E. Gambaro, Diventare autrice, Milano, Unicopli, 2018; D. Brogi, Esiste una letteratura maschile?, «Nazione Indiana», 21 giugno 2015: <https://www.nazioneindiana.com/2015/06/21/esiste-una-scrittura-maschile/>. Per una filosofia femminista della letteratura, A. Cavarero, Tu che mi guardi, tu che mi racconti: filosofia della narrazione, Milano, Feltrinelli, 2011.

5. Sul concetto di intersezionalità e la sua origine nel pensiero femminista nero americano, si veda A. Carastathis, The Concept of Intersectionality in Feminist Theory, «Philosophy Compass», vol. IX, n 5, 2014, pp. 304-314; K. W. Crenshaw, Demarginalizing the Intersection of Race and Sex: A Black Feminist Critique of Antidiscrimination Doctrine, Feminist Theory and Antiracist Politics, «University of Chicago Legal Forum», 1989, pp. 139-167; Id., Mapping the Margins: Intersectionality, Identity Politics, and Violence against Women of Color, «Stanford Law Review», vol. XLIII, n 6, 1991, pp. 1241-1299.

6. Ringrazio la British Academy per il finanziamento del progetto, intitolato «The Gender of Literature. Italian Women Writers and the Literary Canon» (University of Warwick, 2017-2020).

7. Per quanto riguarda il dibattito corrente, si vedano per esempio: L. Spagnol, Maschilismo $e$ letteratura, cosa ci perdiamo noi uomini?, «Il libraio», 11 ottobre 2016; M. Murgia, Cultura maschilista? Dai festival ai giornali, la sottorappresentazione delle donne, «Il libraio», 13 ottobre 2016: $<$ www.illibraio.it/cultura-maschilista-festival-donne-398468/>; la ricca serie di articoli di L. Lipperini sul suo blog «Lipperatura», fra cui Divagazioni sulla lettrice (inchiesta in 5 articoli), 
13 gennaio 2020: <http://loredanalipperini.blog.kataweb.it/lipperatura/2020/01/13/divagazionisulla-lettrice-uno/>; la serie di articoli del dossier Donne e uomini nell'editoria: posizioni, uso e mercato, pubblicato su «InGenere» nel corso del 2018: <www.ingenere.it/dossier/donne-uominieditoria-posizioni-uso-mercato>; A. Bazzoni, Il genere della letteratura (inchiesta in 4 articoli), «La balena bianca», 23 maggio 2016: <https://www.labalenabianca.com/2016/05/23/il-genere-dellaletteratura-la-storia-14/>; J. Bonner, Where Are the Great Italian Women Writers?, «LitHub», 7 giugno 2017: <https://lithub.com/where-are-the-great-italian-women-writers/>; A. Momigliano, "The Ferrante Effect": In Italy, Women Writers Are Ascendant, "The New York Times», 9 dicembre 2019: <www.nytimes.com/2019/12/09/books/elena-ferrante-italy-women-writers.html>.

8. Fra i contributi recenti di più ampio respiro sui rapporti fra scrittrici, pensiero femminista $e$ canone in Italia si vedano per esempio: A. Ronchetti e M.S. Sapegno (a cura di), Dentro/Fuori, Sopra/Sotto. Critica femminista e canone letterario negli studi di Italianistica, Ravenna, Longo, 2007; L. Fortini, Critica femminista e critica letteraria in Italia, «Italian Studies», vol. LXV, $\mathrm{n}^{\circ} 2,2010$, pp. 178-191; V. Cox e C. Ferrari (a cura di), Verso una storia di genere della letteratura italiana, Bologna, Il Mulino, 2011; M. Crispino (a cura di), Oltrecanone. Generi, genealogie, tradizioni, Roma, Iacobelli, 2015; S. Parmegiani e M. Prevedello (a cura di), Femminismo e femminismi nella letteratura italiana dall'Ottocento al XXI secolo, Firenze, Società editrice fiorentina, 2019.

9. Per un'analisi aggiornata dei dati si veda dell'Associazione Italiana Editori, Rapporto sullo stato dell'editoria in Italia, Ediser, 2019.

10. Per un approfondimento sui dati e sui meccanismi di assegnazione dei maggiori premi letterari italiani, si veda L. Junk, I premi raccontano (inchiesta in 5 articoli), «ImPagine», 2018: $<$ www.impagine.it/cultura-2/letteratura/i-premi-raccontano/>.

11. A. Cortellessa, Del XXI secolo il canone è questo, «La Stampa», 11 gennaio 2020: $<$ www.lastampa.it/tuttolibri/2020/01/11/news/del-xxi-secolo-il-canone-e-questo-1.38311746>.

12. Per una ricostruzione storica ampia e precisa della retorica universalista nei giudizi dei critici letterari italiani, fra cui De Sanctis, Cattaneo, Croce e Dionisotti, si veda C. Barbarulli e L. Brandi, La biografia di un'idea, in A. M. Crispino (a cura di), Oltrecanone, cit., pp. 89-108.

13. Sul rapporto conflittuale di Elsa Morante con il femminile e il femminismo, si veda E. Porciani, Eroismo, nazione e femminile in Elsa Morante: un percorso testuale verso «La Storia», "The Italianist», vol. XXXIX, nº 3, 2019, pp. 347-363.

14. A. M. Ortese, Corpo celeste, Milano, Adelphi, 1997, p. 52.

15. N. Ginzburg, Il mio mestiere, in Le piccole virtù, Torino, Einaudi, 1962, p. 82.

16. R. Silvestri, Sicuri che l'editoria italiana valorizzi le donne?, «Corriere della Sera», 25 febbraio 2014, <http://officinamasterpiece.corriere.it/>.

17. Per una critica femminista della normatività del concetto di 'letterarietà' così come ereditato dal canone letterario italiano, si veda: E. Forenza, Materiale / eccedente: dalla «potestas» del canone letterario alla «potentia» delle narrazioni, in Ronchetti e Sapegno, Dentro/Fuori, cit., pp. 65-72. Il nodo delle gerarchie stabilite dal concetto di letterarietà è affrontato secondo una prospettiva sociologica bourdieusiana da V. Spinazzola, La modernità letteraria, Milano, Il Saggiatore, 2001.

18. U. Fanning, The Paratext, in Ead., Italian Women's Autobiographical Writings, cit., pp. 189-214. 


\section{RIASSUNTI}

L'articolo esplora il sistema letterario italiano da un punto di vista di genere attraverso interviste a cinque scrittrici contemporanee: Antonella Cilento, Helena Janeczek, Laura Pugno, Caterina Serra e Nadia Terranova. Le interviste consentono una duplice focalizzazione: da un lato, mirano a far emergere le dinamiche di genere nel campo della letteratura italiana contemporanea secondo la prospettiva interna di chi vi partecipa da vicino; dall'altro, danno voce al posizionamento soggettivo e all'esperienza delle scrittrici stesse, nell'intreccio con la loro personale poetica e postura politica. Le conversazioni ruotano intorno a quattro aree tematiche: l'autorialità; le genealogie e il canone; il rapporto con l'editoria, la critica e il pubblico; il rapporto con il femminismo, e fotografano uno scarto profondo fra la consapevolezza e la libertà autoriali rivendicate dalle scrittrici, da un lato, e un contesto letterario ancora a dominanza maschile e patriarcale, dall'altro.

Cet article explore le système littéraire italien du point de vue du genre à travers des entretiens avec cinq écrivaines italiennes contemporaines: Antonella Cilento, Helena Janeczek, Laura Pugno, Caterina Serra et Nadia Terranova. Les interviews permettent une double focalisation : d'un côté, elles visent à faire émerger les dynamiques de genre dans le domaine de la littérature italienne contemporaine, selon la perspective interne des personnes qui y participent directement; de l'autre côté, elles donnent la parole au positionnement subjectif et à l'expérience des écrivaines elles-mêmes, en lien avec leur poétique personnelle et leur position politique. Les conversations portent sur quatre thémes majeurs : l'auctorialité ; les généalogies et le canon littéraire ; le rapport avec l'édition, la critique et le public ; le rapport avec le féminisme, et font apparaître un écart profond entre, d'un côté, leur conscience et leur liberté comme autrices et, de l'autre, un système toujours marqué par la domination masculine et le patriarcat.

The paper investigates the Italian literary system from a gender perspective, through interviews with five contemporary Italian writers: Antonella Cilento, Helena Janeczek, Laura Pugno, Caterina Serra and Nadia Terranova. The interviews present a double focus: on the one hand, they aim to bring out the gender dynamics in the field of contemporary Italian literature from the internal perspective of the persons who contribute directly to it; on the other hand, they give voice to the subjective attitude and experience of the writers themselves, in connection with their personal poetics and political stance. The conversations centered on four thematic areas: auctoriality; genealogies and the literary canon; the relationship with publishing, criticism and the public; and the relationship with feminism. They reveal a deep gap between, on the one hand, the authorial consciousness and freedom claimed by the women writers, and, on the other hand, the poor recognition of their value, since reception is negatively conditioned by a system still marked by a male-dominated and patriarchal system.

INDICE

Keywords : gender, literary system, reception, contemporary Italian women writers, authorship, feminism

Parole chiave : genere, sistema letterario, ricezione, scrittrici italiane contemporanee, autorialità, femminismo

Mots-clés : genre, système littéraire, réception, écrivaines italiennes contemporaines, auctorialité, féminisme 


\section{AUTORE}

\section{ALBERICA BAZZONI}

ICI Berlin Institute for Cultural Inquiry ORCID: 0000-0001-5115-493X

alberica.bazzoni@ici-berlin.org 